\title{
Lenguaje y cognición en la cosmovisión andina
}

\author{
FÉLix Quesada CAstilio \\ Universidad Nacional Mayor de San Marcos
}

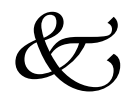

\section{Restmen}

Se analiza la categoría de número en el sistema de persona en las lenguas quechua y aimara. El análisis se sustenta en consideraciones tanto diacrónicas como sincrónicas y el autor evalúa las propuestas inscritas en la lingüística andina. Sobre la base de este análisis, se establece la posible correlación del sistema morfológico con la casmovisión del hombre andino. Al respecto, el autor incide en las sustantivas diferencias de la cognición andina con respecto a la estructura morfológica de una lengua occidental como la castellana.

Palabras claves: Quechua, aimara, cognición, sistema de persona, cosmovisión andina.

\section{Introducción}

T as lenguas andinas quechua y aimara muestran un rasgo Lcaracterístico en cuanto a la categoría de número, distinto con respecto al castellano, lengua occidental. En la teoría gramatical, el número es la categoría gramatical que indica si la palabra se refiere a un dbjeto único (singular) o más de uno (plural) : niño / niños. Mediante esta categoría, los hablantes (del español, por ejemplo) oponen la unidad a la pluralidad, donde número implica noción de cuantificación, multiplicidad dentro de una 'totalidad' . 


\subsection{Persona y número}

El número es una categoría del nombre y se combina de una manera especial con la categoría de la persona para formar pronombres personales (Lyons 1971: 228). La persona es una categoría gramatical que suele ser común al nombre y al verbo. En el verbo esta categoría se refiere a la "calidad de los participantes" (Lyons 1971: 289) . La "primera" persona (Ip) es utilizada por el hablante para referirse a sí mismo como tema del discurso; la "segunda" persona (2p) es utilizada para referirse al oyente o interlocutor y la "tercera" persona (3p) , para referirse a persona o cosa distintas del hablante y el oyente.

\subsection{Persona y número en castellano}

Con respecto a la categoría de número, se distinguen las personas en singular:

$\begin{array}{ll}1 p & \text { como } \\ 2 p & \text { comes } \\ 3 p & \text { come }\end{array}$

Asimismo, estas son las formas para personas en plural:

Ip i comemos

$2 p$ in coméis

3p i comen

Aquí en el plural se expresa multiplicidad de los participantes del discurso frente a la individualidad. En el singular se designa la unidad, mientras que en el plural se indica que "más de uno" (no se especifica la cantidad de miembros) realiza la acción. En este sentido, el plural constituye una totalidad abstracta. 


\subsection{Persona y múmero en quechua y aimara}

En el quechua y el aimara, la categoría de número se manifiesta con la diferencia sustancial que han explicitado todas las gramáticas. Estas lenguas distinguen dos plurales de primera persona que han sido identificados como inclusivo (Incl) y exclusivo (Excl), siguiendo la tradición occidental de la teoría gramatical. Fl Incl es un plural en el cual el oyente u oyentes están incluidos, mientras que el Excl excluye al oyente. El Incl se manifiesta a través de un sufijo -nèik (que presenta variantes dialectales), en tanto que el Excl se forma añadiendo a la marca de primera persona un sufijo pluralizador (-ku, -jiapa, -kuna, -yaa, dependiendo de la variedad dialectal) .

Ia distinción de dos plurales para la primera persona en el quechua y el aimara ha motivado diversas interpretaciones entre los lingüistas.

Pottier (1963) piensa en el Incl debe ser considerado un plural "general" o "universal" que no se inserta en la flexión personal regular, como sí lo hace el "exclusivo".

Martha Hardman (1983) postula en el Incl es una persona diferente y que en la gramática del aimara es necesario considerarlo como cuarta persona, debido a que en el Incl el hablante y oyente están incluidos, frente a las otras personas que no lo están.

Estas hipótesis han sido elaboradas en cada caso partiendo de la asunción de la existencia de un sufijo de carácter atómico que expresa este tipo de plural. Así lo analizan las gramáticas y dentro de los estudios diacrónicas se ha reconstruido el Incl como *-nèik. Nosotros pensamos que este sufijo es analizable en constituyentes; y estos permiten establecer sus componentes semánticos, identificar su función e inferir su significadb altural.

\section{Análisis de paradigma y de -nèik}

Los paradigmas son representantes de cuatro zonas dialectales (Cuadros $N^{\circ} 1$ y 2) . Todos estos dialectos quechuas hacen la distinción entre Incl y Excl y marcan el Incl con -nèik o sus variantes. Este hecho también está presente en el jaqaru y el aimara, lo que indica que es una característica de las lenguas vigentes de la cultura andina. 
2.1. De acuerdo con Solá (1967) y Cusihuamán (1976) -nèik actualmente es analizable en n-èik, donde n- es la tercera persona. Nuestra hipótesis es que este sufijo es segmentable dentro de una perspectiva diacrónica en:

1 èi $k$

Ip Asociativo $2 p$

donde $-k$ sería el segmento velar de la segunda persona -ki y que actualmente esta - $k$ sería lo que queda después del proceso de elisión o pérdida de $i$ ( $-k i>-k)$.

Nuestra hipótesis se sustenta en las siguientes evidencias:

1) La identificación reciente de la forma nèiki en una zona ribereña del Marañón ubicada entre las provincias de Pataz (La Libertad) y Sihuas (Ancash) no estudiada hasta los primeros años de la década del $70^{2}$.

2) Dicha zona muestra la forma y el proceso de caída de $i$, habiéndose registrado las altemancias -nèiki -nèik. Situación similar se da en el quechua de Ferreñafe (Taylor 1982).

\subsection{El sistema en el jaqaru y aimara}

El inclusivo en el aimara y el jaqaru está marcado por -tana (altemado con la variante -tan en el aimara) , el cual estaría compuesto de la $2 p$-ta y -na que indica Ip dado que el pronombre de primera persona /yo/ es naya na en el aimara y na en el jagaru. Es decir, que el aimara y jacaru presentarían el tipo de pluralidad que predice nuestra hipótesis para el quechua, lengua andina como las arriba indicadas. Así, el inclusivo, en tanto que es una forma de pluralidad, expresa una asociación de primera y segunda persona (hablante y oyente) . Nuestra hipótesis, apoyada por la evidencia dialectal del quechua y el sistema del jaqaru y el aimara, nos conduce a la primera conclusión de orden lingüístico: que en el quechua la forma del plural no estaba marcada por un pluralizador, sino por la suma o asociación de 1p y 2p. Esta asociación se establecía mediante el asociativo o asociador, el -èi, el mismo que podría estar vinculado al -èi denominado causativo, el cual cuando se combina con el sufijo -na indica que la acción mutua 
se lleva a cabo con intervención de otro agente. Esta misma asociación ocurre en el jaqaru y el aimara, donde está explícita la asociación de Ip y $2 p$ aunque no se manifiesta formalmente el elemento asociador.

Esta forma asociativa conformada por la Ip + 2p habría constituido el tipo de plural en las etapas más tempranas de la cultura andina. Luego se desarrollarían las otras formas de plural general, como una totalidad, indicadas por el pluralizador -ku o -kuma, al parecer derivado de la forma del recíproco -na y del medio pasivo - ku³, así como las otras formas más recientes. Este fenómeno del quechua está presente, en cierta medida, en el aimara y el jaqaru, en donde la noción de pluralidad como 'masivización' o una 'totalidad' es expresada por -naka (aimara), -kumay -roarya, 'toob' 'toobs' (jacanu).

Esta hipótesis desvirtuaría la tesis de Pottier (1963) , la que sastiene que el Incl en el quechua es un plural totalizador y de carácter universal. Además, nuestra explicación recusa la postulación del Incl en el aimara y el jaqaru como una cuarta persona por parte de Hardman [ (1983), (1986) ] , que es bastante discutible; sin embargo su consideración de que dicho Incl expresa inclusión de Ip y 2p es interesante y apoya en cierto modo la presente tesis.

\section{El correlato aultural}

Ias evidencias lingüŕsticas, sobre toob de orden diacrónico, de que la forma de plural en las lenguas quechuas y aimaras en las etapas más tempranas se indicaba mediante la secuencia de elementos, o asociación de personas, nos lleva a sugerir un posible correlato con la cultura y su desarrollo.

La manera como se indica el plural en las lenguas andinas pude responder al tipo de comunidad y a la concepción de número, y a la manera de operar con éste el mundo andino.

Además del caso Incl, en el quechua existen sufijos o partículas, tanto para nombres como para verbos, que indican pluralidad mediante un procedimiento específico y donde parece estar ausente lo meramente "cuantitativo" y la "masividad"4, como ocurre en la cultura occidental: 
Fínix Quesada Castilio

$\begin{array}{ll}\text {-sapa } & \text { 'aumentativo' } \\ \text {-17apa } & \text { 'completo' } \\ \text { masi } & \text { 'compañero, socio' } \\ \text { pura } & \text { 'entre, con' (grupalizador) }\end{array}$

Entre los sufijos de derivación verbal tenemos los que pluralizan no 'masificando' ni 'totalizando' a los participantes del evento, sino modificando el aspecto de la acción en la siguiente forma:

1 Prolongando la acción.

-nya 'arantitativo' (Cusco)

kuntinyay 'regresar continuamente'

2 Indican que la acción se realiza de manera diferente de lo normal, donde está implicado el número.

triya 'exagerativo' (Cusco, denota que la acción del verbo se realiza de manera desmesurada o no usual)

chuspa-tiya- 'revolcarse' (como un cerdo)

q'api-tiya-y 'manosear torpemente'

-ropari pluralizador (Ancash)

mikurqaarin 'come en cantidad, come en grandes trozos'

kutirgaarin 'estaba regresando varias veces'

3. Pluralidad como secuencia de acciones. La acción del verbo se representa como una serie de acciones o elementos. Las construcciones con los sufijos -paku-, -paya y -ri que, a continuación, presentamos muestran este tipo de pluralidad.

-paaku

(Ancash)

mikrpaakun

'permanece comiendo' = 'come bastante'

-paya frecuentativo y de compañía

much' apayan 'besar varias veces'

puñupayay 'dormir junto a'

-7i (Cajamarca) presenta la cantidad como una secuencia:

rantiriykan 'vuelve a comprar' = 'comprar bastante' 
Se puede reduplicar si la cantidad se incrementa o se multiplica:

rantiririykan 'compra mucho, abundantemente' (reiterativamente)

Estos casos muestran que la pluralidad en la cultura andina no es una mera cuantificación o multiplicación constreñida a una generalización en una totalidad, sino que:

1 El plural básicamente tiene un carácter asociativo, expresado por secuencia o asociación.

2 Esta pluralidad no es exclusivamente cuantitativa; es más bien, caracterizadora y diferenciadora.

3. En este carácter del número está presente la evaluación del hablante. Es el caso de los asociativos y aumentativos aplicados a nombres y verbos. Estos progresivamente han ido perdiendo estas notas y se han constituido actualmente en meros pluralizadores que implican cuantificación. Tal es el caso de -na que en na-ku indica acción recíproca (un tipo de pluralidad) y que en los dialectos quechuas modernos funciona como mero pluralizador. Lo mismo ocurre con sapa y llapa, pues están perdiendo su significado de aumentativo y completivo, respectivamente, y retienen solo el rasgo de "pluralidad". Este fenómeno se ha cumplido en los dialectos quechuas del norte, en los cuales -ijapa (<Ilapa) y -sapa (<*sapa) funcionan como pluralizadbres con el significadb único de 'arantificación' (Torero 1964, 1968 y 1974 ; Quesada 1972 y 1976)

4 La pluralidad se expresa mediante asociación de elementos, secuencia de éstos o acciones que están en la experiencia de los hablantes: sus miembros pueden ser apreciados y evaluados por éste. Tal es el caso de los asociativos cono el Incl, los frecuentativos y repetitivos.

El carácter asociativo y secuencial del plural, como el del Incl, donde sus miembros pueden ser identificados y evaluados, puede estar vinculado al tipo de relación concreta que caracteriza el comportamiento del hombre andino: su comportamiento en la comunidad se cumple mediante relaciones concretas y directas. Por ejemplo, Dios es una realidad concreta con quien se vincula. En las 
lenguas andinas el oyente es muy importante y siempre está presente en el discurso y por eso su participación requiere de una marca especial. En la comunidad andina el interlocutor es alguien que está cerca del hablante, está asociado con éste, con el que interactúa cotidianamente. La tercera persona no aparece y no hay marca morfológica. Esto nos lleva a pensar que la asociación de yoy tú que forman el 'asociativo' identificado como Incl responde al carácter social de las comunidades andinas: su solidaridad.

Ha habido una tendencia a explicar los plurales que implican distintos tipos de relación 1p y 2p, mediante la correlación con estructuras sociales de tipo solidario y no solidario.

La existencia de una forma especial para el plural Incl y la ausencia para el Excl nos conduce a postular una explicación histórica.

Sobre la base de las evidencias disponibles, sostenemos que la categoría de número en el paradigma de persona de las lenguas andinas supérstites constituye un continuum diacrónico: partiendo de la existencia del asociativo (del cual ha derivado el Incl) que debió darse en una etapa temprana en el desarrollo de la sociedad andina y que lingüísticamente correspondería al periodo denominado como preprotoquechua (Quesada 1988) . La evolución del plural en esta lengua pasaría por un periodo en el que se desarrollaría el plural de la primera persona (el Incl y el Excl) como una cuantificación general o como una totalidad. Este nuevo sistema de pluralidad está presente en el periodo llamado protoquechua, dado que lo exhiben la mayor parte de los dialectos quechuas modernos, así como el aimara y el jaqaru. Posteriormente se extendería gradualmente el sistema del plural a las otras personas del paradigma tal como lo testimonian los actuales dialectos quechuas. (CF. Cuadro 1 ).

Finalmente, venoría la etapa de la eliminación del Incl en la que se pierde la distinción entre el Incl y el Excl quedando sólo un plural de primera persona y el paradigma singular y plural semejante al castellano. Es el caso de los dialectos norteños, entre los cuales está el quechua ecuatoriano. 
LENGUAJE Y COGNICIÓN EN LA COSMOVISIÓN ANDINA

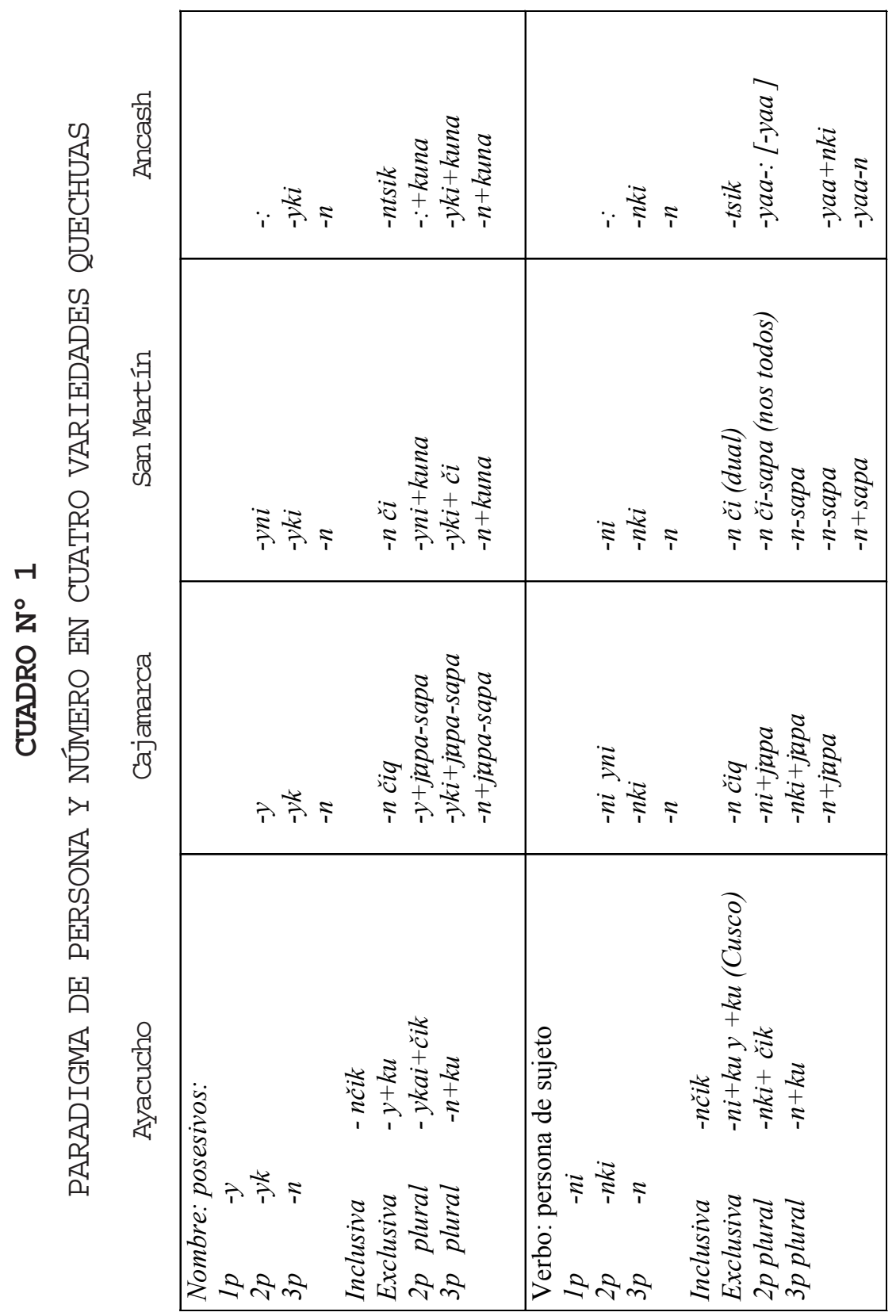




\section{CUADRO $\mathrm{N}^{\circ} 2$ \\ PARADIGMA DE PERSONA EN LAS LENGUAS JAQARU Y AIMARA}

\begin{tabular}{|lll|}
\hline & JAQARU & AIMARA \\
$1 p$ & $-t a " a$ & $-t$ "a ta \\
$2 p$ & $-t a$ & $-t a$ \\
$3 p$ & $-i$ & $-i$ \\
Ird & - tana & $-t a n a(\tan )$ \\
\hline
\end{tabular}

\section{Notas}

1 Ciertas variedades del quechua, como la ecuatoriana, no realizan esta distinción, como resultado de los cambios acontecidos por factores que deben ser identificados.

2 La presencia de -nèiki fue identificada por el autor en 1975 en el noreste de la zona del callejón de conchucos (sección oriental de la provincia de Sihuas-Ancash y parte occidental de la provincia de Pataz, La Libertad), es decir, en las dos riberas del río Marañón (Quesada, 1988). Posteriormente, Taylor (1982) registró esta forma de del Incl en el quechua de Ferreñafe.

3 Cf. el 3, donde se sugiere que el pluralizador del aimara -naka puede ser el resultado de los componentes -na y -ka, fenómeno similar al recíproco en quechua.

4 Este tipo de pluralidad podría estar relacionado con los sistemas de numeración de ciertas culturas amerindias que no generan números por encima de tres o cinco (Gustavo solís, comunicación personal) .

\section{Referencias bibliográficas}

ADEIAAR, W. F. H. (1977) : Tarma Qedhua: Gramar, texts, dictionary. The Peter de Ridder, Lisse. Leiden.

ADEIAAR, W. F. H. (1984) : "Gramatical vowel length and clasification of quechua dialects". Fn IJAL, 50, 1; pp. 25-47. 
BEVER, T. G. (1970) : "The cognitive basis for linguistic structures" . En Cognitition and languaje leaming. Willey, J. R. Heyes editor. NewYork.

BRICGS, L. T. (1976) : Dialectal variation in aimara language of Bolivia and Perú. University of Florida, Gainesville. Doctoral dissentation.

CuSIHUANÁN Gutiérrez A. (1976) : Gramática quedha: Azco-Collao. Ministerio de Educación / Instituto de Estudios Penuanos. Lima.

CERRÓN Palomino R. (1979) : "La primera persona poseedora-actora del protoquedula". En Lexis, 3; pp. 1-39.

CERRón Palomino, R. (1984) : "La reconstrucción del protoquechua" . En Revista Andina, 3; pp. 89-120.

HARDMAN, M. J. (1975) : "Proto-jaqui: reconstrucción del sistema de personas gramaticales". En Revista del Museo Nacional, XII; pp. 434-456.

HARDIMN, M. J. (1983a) : Jaganu: Compendio de estructura fonológica y morfológica. Instituto de Estudios Penuanos. Lima.

HARDINAN, M. J. et al (1986b) : Aymara: Compendio de estructura fonológica y gramatical. IICA. La Paz.

HEIMM, I. H. LASNICKY R. MAY (1991) : "Reciprocity and plurality" . En Linguistic Inquiry, 22, 1: pp. 63-101.

IFNNEBERG, E. (1974) : Nuevas direcciones en el estudio del lenguaje. Gredos. Madrid. IFNERBERG, E. (1975) : Flndamentos biológicos del lenguaje. Alianza Universitaria. Madrid.

LURIA, A. R. (1974) : Ienguaje y comportamiento. Fundamentos. Madrid.

MANHETIM, B. (1982a) : "Anote on 'Inclusive/Exclusive' in sixteen century Perú". En IJAL, 48, 4; pp. 450-459.

MANHEIIM, B. (1982b) : "Person, number and inclusivity in two andean languages". FnActa Lingürística Hefriensia.

NATHIOT, M. (1974) : "La significación cognoscitiva de la categoría del número nominal en papago" . Fn Paul Garviny Yolandra Lastra (editores) Antología de estudios de etrnolingüística y sociolingüística. Instituto de Investigaciones Antropológicas. Universidad Nacional Autónoma de México. México.

PARKER, G. J. (1963) : Derivación verbal en quechua de Ancash. Doamento de trabajo 25. UNMSM-CILA. Lima. 
PARKER, G. J. (1976) : Gramática quechua: Ancash-Hiraylas .Ministerio de Educación e Instituto de Estudios Penuanos. Lima.

POITIFR, B. (1963) : "Inclusif et exclusif dans le sisteme personnel du quechua" . Fn Bulletinde la Faulté des Iettres des Strasbarq. Tilas III.

QUESADA Casti1lo, F. (1976) : Gramática quechua: Cajamarca. cañaris. Ministerio de Educación / IFP. Lima.

QLSADA Casti7lo, F. (1988): Phonological processes in quech a and their implications for the phonological theory. Buffalo, New York State University. Doctoral dissertation.

SANIO TOMÁS, Domingo de, fray (prólogo de Raúl Porras Barrenechea) (1951) : Gramática o arte de la lengua general de los indios de los reynos del Penú. Imprenta Santa María. Edición facsimilar. Lima, 1960.

SAPIR, E. Y Morris SWADEWSH (1974) : "Categorías gramaticales de las lenguas amerindias" . En Paul Garvin y Yolanda Lastra (editores) Antología de estudios de etnolingüística y sociolingüística. Universidadnacional Autónona de Néxico, Instituto de Investigaciones Antropológicas. Néxico.

SOLÁ, D. F. (1967) : Gramática del quechua de Hháruco. UNMSM-PFL. Lima.

SOIO Ruiz, C. (1976) : Gramática quechua: Ayaaudho-chanca. Ministerio de Educación IFP. Lima.

TAYIOR G. (1982) : "Breve presentación de la morfología del quechua de Ferreñafe". Lexis, 6, 2; pp. 243-270.

TORERO, A. (1964) : "Los dialectos queduas". En Anales Científicos de la Lniversidad Agraria, 2; pp. 446-478.

TORERO, A. (1968) : "Procedencia geográfica de los dialectos quechuas de Ferreñafe y Cajamarca". Fn Anales Científico de la UniversidadAgraria, 3-4; pp. 291-16.

TORERO, A. (1974) : El quedira y la historia social andina. Universidad Ricardo Palma, Lima. 